\title{
A Comparative Study of Detection and Classification of Emotions on Social Media Using SVM and Näive Bayes Techniques
}

\author{
Aysha $\mathrm{A}^{\mathrm{a}, 1}$, Syed Meeral $\mathrm{MK}^{\mathrm{a}}$ and Bushra $\mathrm{KM}{ }^{\mathrm{a}}$ \\ ${ }^{a}$ Department of Computer Science, JBAS College for Women, Teynampet, Chennai, \\ Tamilnadu, India
}

\begin{abstract}
The rapid rate of innovations and dynamics of technology has made humans life more dependent on them. In today's synopsis Microblogging and Social networking sites like Twitter, Facebook are a part of our lives that cannot be detached from anyone. Through these social media each one of them carry their emotions and fix their opinions based on a particular situations or circumstances. This paper presents a brief comparison about Detection and Classification of Emotions on Social Media using SVM and Naïve Bayesian classifier. Twitter messages has been used as input dataset because they contain a broad, varied, and freely accessible set of emotions. The approach uses hash-tags as labels to train supervised classifiers to detect multiple classes of emotion on potentially large data sets without the need for manual intervention. We look into the usefulness of a number of features for detecting emotions, including unigrams, unigram symbol, negations and punctuations using SVM and Naïve Bayesian Classifiers.
\end{abstract}

Keywords. Emotions, Microblog, Twitter, SVM, Naïve Bayes.

\section{Introduction}

Social media are interactive digitally mediated technologies that facilitate the creation or sharing or exchange of information, ideas, career interests, product reviews, and other forms of expression via virtual communities and networks. The word "emotion" was coined in the early 1800s by Thomas Brown and it is around the 1830s that the modern concept of emotion first emerged for the English language. With the advent of social networking platforms, communication has shifted from face-to-face to online with instant messaging, a means of communication that involves a lot of communication using unigram symbols and short text types. In texts, text-based representations of emotions are used. The opinion of other person is an important information for decision making. It also needs multi-label classification [1]. The information from Social Medial is used to organize, explore and analyse for better decision making. Even though the study of

\footnotetext{
${ }^{1}$ Aysha A, Department of Computer Science, JBAS College for Women, Teynampet, Chennai.

E-mail: aysha.a@jbascollege.edu.in.
} 
emotion dates back to the 1980 s, emotion computation is a relatively new field. With so much data available from social media, the role of classification is complicated by issues such as the lack of a common dataset for lexical analysis of the data. Annotating the data has its own set of problems, such as the possibility of bias if a human expert is used, and the difficulty of gathering annotated data from online resources. Emotion analysis on Twitter data has a duration issue because it articulates the emotions in a short amount of time, and the increasing amount of slang content increases the amount of preprocessing needed. Automating machines to detect emotions will help many organizations to understand their consumers effectively and to make more appropriate decisions. This study exhibits the trends in the emotion recognition in different perspectives. Even there is many techniques are there to exhibit the emotion recognition still the machines need some efficient learning algorithm to provide the result thinking as human. An overview of how the emotions is detected can be seen in Figure 1.

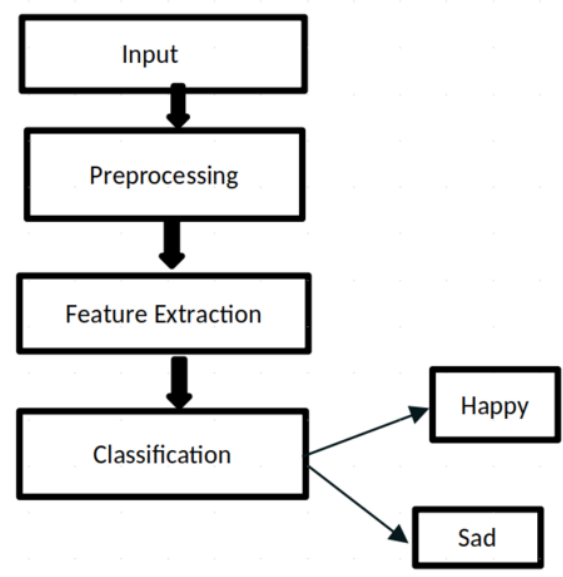

Figure 1. Flow diagram for emotion detection

\subsection{Social Media: Twitter}

Emotion is a combinatorial result of a person's evaluation of a situation along with the physiological arousal. One of the earliest studies suggests 'anger', 'fear', 'enjoyment', 'sadness', 'disgust', 'surprise' as the basic emotions [2]. Individuals can share their views, emotions, and thoughts on a variety of topics in the form of short text messages using social networks and microblogging tools like Twitter. These are brief texts (commonly known as tweets) where individuals mental states (such as pleasure, anxiety, confusion, depression) as well as the feelings of a wider group reflecting various forms of emotions (such as opinions of people in a certain country or affiliation). In reality, Twitter can be thought of as a vast repository containing a diverse range of emotions, feelings, and moods [3]. For example, the tweet "Happy birthday brother, wish $u$ all the health, happiness, success. Stay blessed Stay safe." Expresses Happiness. "I am lost. My life is broken" expresses sadness as shown in Table 1.

The role of emotion analysis and classification using machine learning techniques [4] (SVM and Naïve Bayes) is the subject of this paper. The information used came from 
Twitter. The data was collected automatically using emotion hashtags for two emotion types which includes Happy and Sad whereas Fear, Stressed, Depressed comes in the indirect state of Sad and Relaxed comes in the indirect state of Happy as given in Table 2 .

Table 1. Examples for emotion

\begin{tabular}{|l|l|}
\hline Happy & Weekend is coming! \\
\hline Sad & RIP! Grandfather passed away. \\
\hline Fear & Everybody dies in theirNightmare. \\
\hline Relaxed & I feel so at peace right now. The sound of rain always puts me to Sleep. \\
\hline Stressed & Seriously! Stressed over this final Presentation? \\
\hline Depressed & My cat is lost. I 'm totally depressed. \\
\hline
\end{tabular}

\subsection{Inducement}

The study demonstrates a method for automatically detecting and classifying the emotions conveyed in Twitter messages. A framework based on this approach could be used in a wide range of applications, including Politics, Entertainment, Health-Care, Transportation, Communications Industry to detect the emotions of group of people and their stature of mind. Self-reports and surveys are commonly used to assess quality of life. People are asked to complete questionnaires about their daily lives and emotions. It takes a long time, is repetitive, and is prone to errors to collect these questionnaires. The framework developed based on studied method, on the other hand, would be able to automatically detect how people feel about their lives from tweets.

\section{Data Pre-Processing}

There will always be noisy, outdated, and meaningless data in any dataset that needs to be cleaned up. Failure to do so makes the training process extremely difficult, resulting in poor classifier results. Data pre-processing is a crucial step in achieving high precision from the noisy data to the best possible data used for training. Pre-processing a typical political reviews tweet dataset. involves a number of techniques. This involves maintaining a consistent letter case, stripping special symbols typically used in tweets like “@,\#,etc.," eliminating extra whitespace and redundant terms, and many such steps [5].

\subsection{Detection of Emotions}

Detection of emotions can be done by Supervised Machine Learning to identify emotions in text messages such as tweets to classify short texts automatically according to the category of the text defined $[3,6]$.

\subsection{Collection of Data}

To pre-process the Twitter message the following rules are taken into consideration. 
1. Tweets often contain usernames which start with the @ symbol before the username (e.g. @Fathima). All words that start with the @ symbol are replaced by"USERID” [5].

2. Many tweets have multiple hash tags, and some even have hash tags from two different classes. For example, the tweet "Iam Employed.. \#nervous \#sohappy." Contains the hash-tag \#nervous from the Sad class and the tag \#sohappy from the Happy class. If there are two subjects in a tweet, it may be omitted because such tweets will add ambiguity as given in Table 2 .

\subsection{Feature preferences}

\subsubsection{Unigrams}

Unigrams define single word that are widely used in expressing emotions directly. The appearance of affect terms such as "disgusted" and "happy" in text messages can be used to classify them into emotion groups as sad class and happy class. Identifying an acceptable emoticon will also solve the problem of detection.

\subsubsection{Unigram Symbols}

Unigram Symbols are textual representations of a writer's emotion in the form of symbols, which are useful features for emotion classification in text messages. In emotion analysis, these features are often used. The western style emoticons were used by Go et al [7] and A. Pak et al [8], to collect labelled data. There are a variety of emoticons that can represent joyful, sad, irritated, or sleepy emotions listed in Table 3.

Table 2. Unigram Emoticons

\begin{tabular}{|c|c|}
\hline Category & Unigram Emoticons \\
\hline Happy & 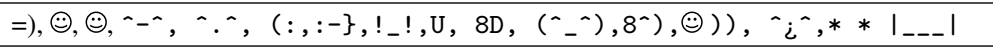 \\
\hline $\mathrm{Sad}$ & 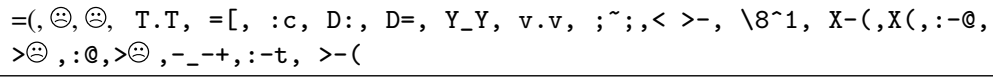 \\
\hline
\end{tabular}

\subsubsection{Punctuations}

Punctuations are another function that may be useful in detecting emotions. When users want to convey their intense emotions, they often use exclamation marks. For example, the tweet "I lost 4lb in 3 days!!!!!" conveys great joy, while the tweet "we're in December, which means... ONE MONTH IS THERE TO GET SALARY!!!” conveys a high level of anxiety. The exclamation mark can be used in a variety of situations.

\subsubsection{Negation}

Negation are the final function to correct errors caused by tweets that contain negated phrases such as "not sad" or "not happy." For example the tweet, "Finally Exams are over .Long wait !.Not happy. Even though it has a cheerful unigram, "Not happy" should be considered a sad tweet. We define negation as a distinct function to address this problem. 


\section{Data Classification}

Machine Learning model learns from the past input data and makes future predictions as output. Classification is a supervised machine learning model that learns data from a classified trained set and predicts the test set without knowing the actual class labels. Decision Trees, Naïve Bayes, Support Vector Machines, k-Nearest Neighbors, Rule based classifier and many more other classification models are among the options. A study on two well-known supervised learning classifiers such as Nave Bayes and Support Vector Machines were demonstrated.

Table 3. Hash Tags Emotions

\begin{tabular}{|l|l|}
\hline Class & Hash-Tags \\
\hline Happy & $\begin{array}{l}\text { \#elated, \#overjoyed, \#enjoy, \#excited, \#proud, \#joyful, \#feelhappy,\#sohappy, \#veryhappy, } \\
\text { \#happy, \#superhappy, \#happytweet, \#feelblessed,\#blessed, \#amazing, \#wonderful, \#excelent, } \\
\text { \#delighted, \#enthusiastic, \#calm,\#calming, \#peaceful, \#quiet, \#silent, \#serene, \#convinced, } \\
\text { \#consent,\#contented, \#contentment, \#satisfied, \#relax, \#relaxed, \#relaxing, \#sleepy,\#sleepyhead, } \\
\text { \#asleep, \#resting, \#restful, \#placid }\end{array}$ \\
\hline $\begin{array}{l}\text { \#nervous, \#anxious, \#tension, \#afraid, \#fearful, \#angry, \#annoyed,\#annoying, \#stress, \#dis- } \\
\text { tressed, \#distress, \#stressful, \#stressed, \#worried,\#tense, \#bothered, \#disturbed, \#irritated, \#mad, } \\
\text { \#furious \#sad,\#ifeelsad,\#feelsad, \#sosad, \#verysad, \#sorrow, \#disappointed, \#supersad, \#miser- } \\
\text { able,\#hopeless, \#depress, \#depressed, \#depression, \#fatigued, \#gloomy, \#nothappy, \#unhappy, } \\
\text { \#suicidal, \#downhearted, \#hapless, \#dispirited }\end{array}$ \\
\hline
\end{tabular}

Naïve Bayes is a probabilistic classifier based on Bayes theorem, with the naïve assumption of independence between every pair of features. This model works well on text classification that are based on posterior probabilities generated with the presence of different classes of words in text form from Twitter. Naive Bayes has been widely used for classifying text because it is simple and fast [9]. A Support Vector Machine (SVM) is a discriminative classifier defined by a separating hyper plane as shown in Figure 3. From the given labeled training data SVM finds a hyper-plane that creates a boundary between the types of data. The two results of a binary classifier will be

- The data point belongs to that class OR

- The data point does not belong to that class.

The best hyper plane is the one that represents the largest separation or margin between the two classes. Text classification using SVMs is very robust to outliers and does not require any parameter tuning [10].

\section{Experimental Results}

\subsection{Identification of Emotion Hash-tags}

The collected labeled data are identified from the list of hash-tags corresponding to each class of emotions. Two classes of emotions namely Happy and Sad are defined. We selected those keywords which are very distinct and distinguishable from other classes. We ignored the keywords that are located close to the boundary of two dimensions. we extended the list by adding hash-tags from Twitter, namely emotion-specific tags such as the tag "\#ifeelsad". Using the extended list of keywords, we obtained a set of unique 


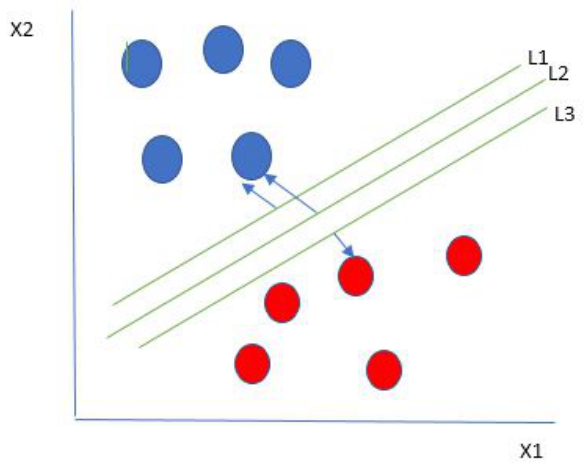

Figure 2. SVM binary classifier

emotion hash-tags for each class. Table 2 presents the final list of hash-tags used for collecting labeled data for each class. Table 4 represents the number of collected labeled tweets before and after pre-processing. As it shows the number of tweets decreased by $19 \%$ by removing noisy tweets during preprocessing.

Table 4. Collected Labelled tweets

\begin{tabular}{|l|c|c|}
\hline Emotions & No.of Tweets before Preprocessing & No.of Tweets before Preprocessing \\
\hline Happy & 80600 & 63200 \\
\hline Sad & 84700 & 70900 \\
\hline Total & 165300 & 134100 \\
\hline
\end{tabular}

Once the pre-processing is performed, each classifier is trained after which the classifiers are individually evaluated on the test samples. Evaluation metrics are used to measure the quality of the statistical or machine learning model. There are many different types of evaluation metrics available to test a model. These include classification accuracy, logarithmic loss, confusion matrix, and others. The test set is a set of observations used to evaluate the performance of the model using some performance metric. In our study three metrics (precision, recall and f-measure) are used to evaluate the performance for both happy and sad tweets.

Precision is also called positive predicted value is the fraction of relevant instances among the retrieved instances. An algorithm with high precision returns more pertinent results as compared to irrelevant results. Recall is a measure that tells us how great our model is when all the actual values are positive. If Recall $=0$, this means that the model is broken. If Recall $=1$, this means that the model is good enough to correctly make a prediction. It is a quantity measure to check how many relevant results are being returned by the algorithm while precision checks from the selected items, how many of them were relevant. F-Measure: F-Measure is a metric that combines recall and precision by taking their harmonic mean.

\subsection{Results of Classification}

In our study we found that $75 \%$ of collected data is labeled data which is used to train a classifier and $25 \%$ for testing. The accuracy of classifiers is measured based on F- 
measure precision and recall. Table 5 present F-measure, precision and recall of SVM and Naive Bayes techniques using different kinds of features. From the table it is understood the accuracy is higher for Naive Bayes for all the features. However SVM achieved the highest accuracy by using unigrams and negations. The accuracy of Naive Bayesian

Table 5. Measures of SVM, Naive Bayes Classifier for different features

\begin{tabular}{|l|c|c|c|c|c|c|}
\hline \multirow{2}{*}{ Features } & \multicolumn{2}{|c|}{ F-Measure } & \multicolumn{2}{c|}{ Precision } & \multicolumn{2}{c|}{ Recall } \\
\cline { 2 - 7 } & Naïve Bayes & SVM & Naïve Bayes & SVM & Naïve Bayes & SVM \\
\hline Unigram & 86.2 & 90 & 87.7 & 90.3 & 86.4 & 89.7 \\
\hline unigram symbols & 86.4 & 89 & 87.5 & 89.5 & 86.3 & 88.8 \\
\hline Punctuations & 86.2 & 89.8 & 87.2 & 90.4 & 86.6 & 89.4 \\
\hline Negations & 86.9 & 89 & 87.9 & 89.4 & 86.9 & 88.7 \\
\hline Total & 86.43 & 89.45 & 87.58 & 89.9 & 86.55 & 89.15 \\
\hline
\end{tabular}

Classification and SVM classification and are presented in Figure 3 and 4. For the class sad the highest accuracy can be achieved by using all the features. However for other classes the highest accuracy can be achieved by using unigrams. Interestingly, using punctuation features across these two classes increased the accuracy.

In this paper, we have studied how to classify Twitter messages into distinct emotional classes based on the Twitter hash-tags. Our results suggest that hash-tags and other conventional markers of tweets are useful features for emotion classification. In the field

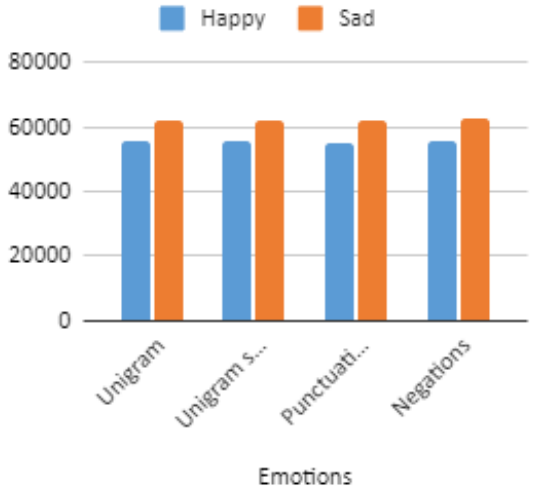

Figure 3. Emotion classification using Naïve Bayes

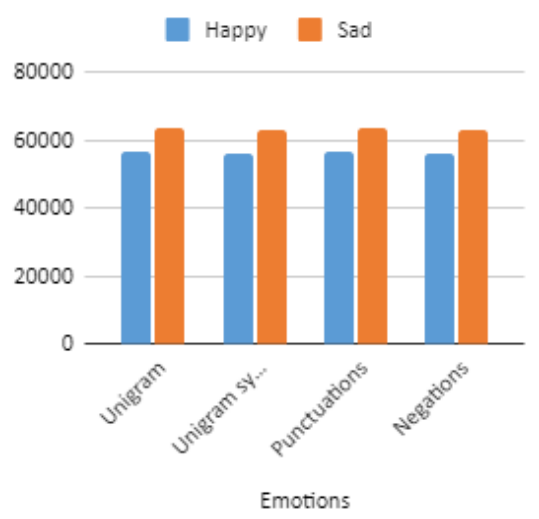

Figure 4. Emotion classification using SVM

of emotion identification, database choice played a pivotal role where feature extraction is the second step for good exactness in the result. From our study, we found that the Naïve Bayes Classification and Support Vector Machines are the most widely used ML algorithms for solving Emotion Classification. Support Vector Machines Classification algorithm is widely used in most of the paper because it is fast and performs better than the other technique $[11,12]$. Still, there are many open issues that need to be solved like diversity in emotion, recognize spontaneous emotion and speaker recognition in case of simultaneous conversation. Tools for Downloading and Analyzing Twitter Data [13], Twitter's official archive download, BirdSong Analytics, Cyfe, NodeXL and TWChat. 


\section{Conclusion and Future Work}

This paper gives a summarized review of the thorough comparison and performance analysis of the salient classification algorithms used in supervised learning namely Naïve Bayes, Support vector machine. We have considered only SVM and Bayesian Classifier because they achieved more accuracy in many studies and even though few other classifiers provide high accuracy, it is very slow and therefore not practical for big datasets. From our standard reviews on tweet dataset, we can say that SVM run fast than Naïve Bayes where the accuracy is $90 \%$ when the accuracy of Naïve Bayes is $87 \%$. As a future work, based on data accessibility, we have a plan to propose method that can be implemented on social media data like Twitter, Facebook etc., Also the proposed method can be used to analyze the emotions on political reviews, disaster management and scholastic review on higher education. Probabilistic, non-probabilistic, and ensemble classifiers are popular in machine learning.

\section{References}

[1] Sabena S, Kalaiselvi S, Anusha B, Ramesh LS. An Multi-Label Classification with Label Correlation. Asian Journal of Research in Social Sciences and Humanities. 2016;6(9):373-86.

[2] Bollen J, Mao H, Pepe A. Modeling public mood and emotion: Twitter sentiment and socio-economic phenomena. In Proceedings of the international AAAI conference on web and social media 2011 Jul 5 (Vol. 5, No. 1).

[3] Sadhana SA, SaiRamesh L, Sabena S, Ganapathy S, Kannan A. Mining target opinions from online reviews using semi-supervised word alignment model. In 2017 Second International Conference on Recent Trends and Challenges in Computational Models (ICRTCCM) 2017 Feb 3 (pp. 196-200). IEEE.

[4] Liu M. Optimization of marine biological sediment and aerobics training mode based on SVM. Arabian Journal of Geosciences. 2021 Aug;14(15):1-7.

[5] Sahu S, Rout SK, Mohanty D. Twitter sentiment analysis-a more enhanced way of classification and scoring. In 2015 IEEE international symposium on nanoelectronic and information systems 2015 Dec 21 (pp. 67-72). IEEE.

[6] Sulthana AR, Jaithunbi AK, Ramesh LS. Sentiment analysis in twitter data using data analytic techniques for predictive modelling. In Journal of Physics: Conference Series 2018 Apr 1 (Vol. 1000, No. 1, p. 012130). IOP Publishing.

[7] Go A, Bhayani R, Huang L. Twitter sentiment classification using distant supervision. CS224N project report, Stanford. 2009 Dec;1(12):1-6.

[8] Pak A, Paroubek P. Twitter as a corpus for sentiment analysis and opinion mining. In LREc 2010 May 17 (Vol. 10, No. 2010, pp. 1320-1326).

[9] Krishnan H, Elayidom MS, Santhanakrishnan T. Emotion detection of tweets using naive bayes classifier. Emotion. 2017 Nov;4(11):457-62.

[10] Joachims T. Text categorization with support vector machines: Learning with many relevant features. In European conference on machine learning 1998 Apr 21 (pp. 137-142). Springer, Berlin, Heidelberg.

[11] Jin Q, Li C, Chen S, Wu H. Speech emotion recognition with acoustic and lexical features. In 2015 IEEE international conference on acoustics, speech and signal processing (ICASSP) 2015 Apr 19 (pp. 4749-4753). IEEE.

[12] Zheng WQ, Yu JS, Zou YX. An experimental study of speech emotion recognition based on deep convolutional neural networks. In 2015 international conference on affective computing and intelligent interaction (ACII) 2015 Sep 21 (pp. 827-831). IEEE.

[13] Ann S. Five Tools for Downloading and Analyzing Twitter Data [Internet]. [updated 2015 Feb 17; cited 2020 Sep 12]. Available from: https://www.entrepreneur.com/article/242830. 